\title{
HOLONOMÍA Y SU RELACIÓN CON LAS FOLIACIONES
}

\section{Teodoro Sulca Paredes ${ }^{1}$}

\begin{abstract}
RESUMEN.- Consideremos campos vectoriales no lineales holomorfos $Z$ definidos en un abierto $U$ de $\mathbb{C}^{2}$ con singularidad aislada en el origen, y se estudia el sistema de ecuaciones diferenciales asociado al campo vectorial $Z$, para el caso en que la parte lineal de dicho campo está en el dominio de Siegel. Se hace uso del concepto de holonomia.
\end{abstract}

PALABRAS CLAVE.- Foliación, campos vectoriales, holonomias, levantamientos.

\section{HOLONOMY AND IT RALATION WITH THE FOLLATIONS}

\begin{abstract}
We consider non-linear holomorphic vector fields $Z$ defined in an open $U$ of $\mathbb{C}^{2}$ with isolated singularity at the origin. We study a system of differential equations associated to the vector field $Z$, for the case in which the linear part of this field is in Siegel's domain. The concept of holonomy is used.
\end{abstract}

KEYWORDS: Foliations, vector fields, holonomy, liftings.

\section{INTRODUCCIÓN}

El caso más simple del presente estudio es cuando el campo vectorial $Z$ es lineal, es decir de la forma

$$
Z\left(z_{1}, z_{2}\right)=\left(\lambda_{1} z_{1}, \lambda_{2} z_{2}\right)
$$

donde la foliación inducida por el campo depende de los números complejos $\lambda_{1}$ y $\lambda_{2}$, surgiendo así los conceptos de Dominio de Poincaré, Dominio de Siegel y Resonancias.

Cuando $Z$ es más general, tal que su parte lineal está en el Dominio de Poincairé y no hay resonancias existe una conjugación analítica local con su parte lineal (Teorema de Poincaré) (Benazic [1]) y cuando existen resonancias

\footnotetext{
${ }^{1}$ Profesor de la Facultad de Ciencias Matemáticas de la Universidad Nacional Mayor de San Marcos. E-mail: tsulcap@unmsm.edu.pe
} 
tenemos una conjugación analítica del campo con una pequeña perturbación de su parte lineal (Teorema de Dulac) (Ramírez [7]).

El método aplicado para la justificación de los resultados mencionados falla para el caso del Dominio de Siegel, por tanto nuestro objetivo es ver lo que sucede en éste caso, para ello usaremos el concepto de holonomia, con lo cual determinaremos cuando dos campos vectoriales son conjugados.

\section{PRELIMINARES}

Sean $U \subset \mathbb{C}^{2}$ abierto. Un campo vectorial $Z$ en $U$ es una aplicación que a cada $z \in U$ le asocia un elemento $Z(z) \in T_{z} \mathbb{C}^{2}$ el espacio tangente a $\mathbb{C}^{2}$ en el punto $z$. Podemos denotar una base de $T_{z} \mathbb{C}^{2}$ por

$$
\left\{\frac{\partial}{\partial z_{1}}(z), \frac{\partial}{\partial z_{2}}(z)\right\}
$$

y con ello podemos escribir

$$
Z(z)=\sum_{j=1}^{2} Z_{j}(z) \frac{\partial}{\partial z_{j}}(z), \forall z \in U
$$

En adelante, escribiremos un campo vectorial como

$$
Z=\sum_{j=1}^{2} Z_{j} \frac{\partial}{\partial z_{j}}, \text { o simplemente } Z=\left(Z_{1}, Z_{2}\right)
$$

Se dice que $Z$ es un campo vectorial holomorfo en $U$ si y sólo si sus funciones coordenadas $Z_{j}$ son holomorfas, en éste caso en cada punto $z_{0} \in U$ existe una vecindad abierta $V, z_{0} \in V \subset U$ tal que $Z_{j}$ tiene expansión en serie de potencias

$$
Z_{j}(z)=\sum_{|Q|=0}^{\infty} b_{j, Q}\left(z-z_{0}\right)^{Q}
$$

los cuales son convergencias para cualquier $z \in V$.

En la relación (1) se han usado las notaciones usuales de los multiíndices, es decir 


$$
\begin{gathered}
Q=\left(q_{1}, q_{2}\right) \in \mathbb{N}^{2} \\
|Q|=\sum_{j=1}^{2} q_{J} \quad y \quad z^{Q}=z_{1}^{q_{1}} \cdot z_{2}^{q_{2}}
\end{gathered}
$$

donde $z=\left(z_{1}, z_{2}\right) \in \mathbb{C}^{2}$.

\section{Definición}

- $\quad z_{0}$ es un punto singular de $Z$ si y sólo si $Z\left(z_{0}\right)=0$; en caso contrario $\left(Z\left(z_{0}\right) \neq 0\right)$ decimos que $z_{0}$ es un punto regular de $Z$.

- $z_{0} \in U$ es una singularidad aislada de $Z$ si existe una vecindad abierta $V$, $z_{0} \in V \subset U$, tal que, cualquier $z \in V, z \neq z_{0}$ es un punto regular.

- A cada campo vectorial holomorfo $Z$ le asociamos el sistema de ecuaciones diferenciales ordinarias:

$$
\left\{\begin{array}{l}
\frac{d z_{1}}{d T}=Z_{1}(z) \\
\frac{d z_{2}}{d T}=Z_{2}(z)
\end{array} \quad, \quad z=\left(z_{1}, z_{2}\right) \in \mathbb{C}^{2}, T \in \mathbb{C}\right.
$$

Por el teorema de existencia y unicidad de las soluciones de las ecuaciones diferenciales ordinarias (Hirsch - Smale [4], Sotomayor [8]), las soluciones de (2) son curvas complejas localmente parametrizadas por $T \in \mathbb{C}$. Estas curvas definen una foliación $\mathcal{F}_{z}$ (Camacho [2]) y cada una de ellas será llamada hojas de la foliación $\mathcal{F}_{z}$.

En el caso lineal las hojas son transversales a los toros $T_{R}:\left|x_{1}\right|=R<R_{0}$. Algo análogo sucede cuando el campo es no lineal de modo que debemos analizar el campo de líneas inducido en $T_{R}$. Como $\left|x_{1}\right|=R, x_{2}=0$ es una curva integral de este campo, entonces su aplicación de primer retorno $h$, está bien definida en el disco $\left\{\left|x_{1}\right|=R,\left|x_{2}\right|<\gamma\right\}$ para $\gamma>0$ suficientemente pequeño.

\section{Definición.}

La aplicación hes denominada holonomía de la solución $x_{2}=0$ en el punto $(R, 0)$. 


\section{RESULTADO PRINCIPAL}

Uno de los resultados trazados en el presente trabajo fue obtener una relación entre holonomías y foliaciones, asi como determinar cuando dos campos vectoriales resultan ser conjugados. El siguiente es el principal resultado del trabajo.

\section{Teorema.}

Sean

$$
Z_{j}=\left(\alpha_{1}^{j} x_{1}+x_{1} x_{2} a_{1}^{j}\left(x_{1}, x_{2}\right)\right) \frac{\partial}{\partial x_{1}}+\left(\alpha_{2}^{j} x_{2}+x_{1} x_{2} a_{2}^{j}\left(x_{1}, x_{2}\right)\right) \frac{\partial}{\partial x_{2}}
$$

campos vectoriales analíticos definido en una vecindad de $(0,0) \in \mathbb{C}^{2}$, con $\left(\alpha_{1}^{j}, \alpha_{2}^{j}\right) \in D_{s}$ y $\frac{\alpha_{1}^{1}}{\alpha_{2}^{1}}=\frac{\alpha_{1}^{2}}{\alpha_{2}^{2}}=\lambda \quad(j=1,2)$. Indiquemos con $h_{j}$ las holonomías respectivas de $x_{2}=0$ en el punto $(1,0)$. Si existe un difeomorfismo local $\xi$ de $\mathbb{C}$ en $0 \in \mathbb{C}$ de modo que $h_{2} \circ \hat{\xi}=\hat{\xi} \circ h_{1}$, entonces existe en difeomorfismo local $\xi$ de $\mathbb{C}^{2}$ en $(0,0) \in \mathbb{C}^{2}$ que transforma la foliaciones asociada a $Z$ en aquella de $Z_{2}$.

\section{Demostración.}

Analizaremos primero para el caso de un campo no lineal y el otro lineal. Sea el campo no lineal

$$
Z_{1}=\left(\alpha_{1} x_{1}+x_{1} x_{2} a_{1}\left(x_{1}, x_{2}\right), \alpha_{2} x_{1}+x_{1} x_{2} a_{2}\left(x_{1}, x_{2}\right)\right)
$$

con $a_{1}(0,0)=0, a_{2}(0,0)=0$ definido en una vecindad de $(0,0) \in \mathbb{C}^{2}$, el cual puede ser transformado en

$$
\tilde{Z}_{1}=\left(x_{1}, \lambda x_{2}\left(1+A\left(x_{1}, x_{2}\right)\right)\right)
$$

con $A(0,0)=0 . \quad($ Camacho - Sad [3]).

Por otro lado, denotemos el campo lineal por $Z=\left(y_{1}, \lambda y_{2}\right)$. El flujo de $\tilde{Z}_{1}$ se obtiene resolviendo el sistema,

$$
\left\{\begin{array} { l } 
{ \dot { x } _ { 1 } = x _ { 1 } } \\
{ \dot { x } _ { 2 } = \lambda x _ { 2 } ( 1 + A ( x _ { 1 } , x _ { 2 } ) ) }
\end{array} , \text { luego } \left\{\begin{array}{l}
x_{1}(T)=e^{T} x_{1} \\
x_{2}(T)=\varphi_{2}\left(T,\left(x_{1}, x_{2}\right)\right)
\end{array}, T \in \mathbb{C} .\right.\right.
$$


Denotemos por $\varphi\left(T,\left(x_{1}, x_{2}\right)\right)$ al flujo que pasa por el punto $\left(x_{1}, x_{2}\right)$, es decir

$$
\varphi\left(T,\left(x_{1}, x_{2}\right)\right)=\left(e^{T} x_{1}, \varphi_{2}\left(T,\left(x_{1}, x_{2}\right)\right)\right) .
$$

En forma análoga, denotamos el flujo de $Z$ por

$$
\psi\left(T,\left(y_{1}, y_{2}\right)\right)=\left(e^{T} y_{1}, e^{\lambda T} y_{2}\right)
$$

Se tiene por hipótesis que $h_{1}$ es holonomía respecto de $x_{1}=0$ en el punto $(1,0)$ y $h_{2}$ respecto a $y_{2}=0$ en el punto $(1,0)$ siendo $\hat{\xi}$ un difeomorfismo local de $\mathbb{C}$ en $0 \in \mathbb{C}$ de modo que,

$$
\begin{aligned}
& h_{2} \circ \hat{\xi}=\hat{\xi} \circ h_{1} \\
& \hat{\xi}:\{1\} \times D_{r_{1}}(0) \mapsto\{1\} \times D_{r_{2}}(0) \\
&\left(1, x_{2}\right) \quad \mapsto\left(1, x_{2}\right)=\left(1, \hat{\xi}\left(x_{2}\right)\right) .
\end{aligned}
$$

Queremos extender $\hat{\xi}$ a

$$
\tilde{\xi}: \mathbb{S}^{1} \times D_{1}(0) \rightarrow \mathbb{S}^{1} \times D_{1}(0) .
$$

Supongamos que $\left|A\left(x_{1}, x_{2}\right)\right|<1 / 2, \forall\left(x_{1}, x_{2}\right) \in D_{1}(0) \times D_{1}(0)$ y que el difeomorfismo de la holomonía $h$ correspondiente al lazo $\gamma:[0,1] \rightarrow L_{0}, \gamma(\theta)=\left(e^{2 i \pi \theta}, 0\right)$ es la rotación $h: y \rightarrow e^{2 i \pi \lambda} y$.

La extensión de $\hat{\xi}$ a $\tilde{\xi}$ lo haremos por el método clásico de levantamiento de caminos (Mattei-Moussu [6]) contenidos dentro de la hoja $L_{0}\left(L_{0}=\mathbb{S}^{1} \cap\left\{x_{2}=0\right\}\right)$ en caminos dentro de las hojas de $\mathcal{F}_{\tilde{Z}_{1}}\left(\circ \mathcal{F}_{Z}\right)$ siguiendo la proyección $(x, y) \rightarrow x$.

El difeomorfismo de la holonomia $h_{1}$ de $L_{0}$ en $(1,0)$ es una rotación, asi existe $\rho>0$ tal que la hoja $F_{\tilde{z}_{1} \mid \mathbb{S}^{1} \times D_{1}}$ que pasa por $\left(1, x_{2}\right)$ para $\left|x_{2}\right|<\rho$ está contenida en $\mathbb{S}^{1} \times D_{1}$. La unión $V_{\rho}$ de esas hojas es una vecindad abierta de $\mathbb{S}^{1} \times\{0\}$ dentro de $\mathbb{S}^{1} \times D_{1}$.

El camino

$$
\begin{aligned}
\bar{\alpha}_{0}: \mathbb{R} & \rightarrow L_{0} \\
\theta & \mapsto \bar{\alpha}_{0}(\theta)=\left(e^{i \theta}, 0\right)
\end{aligned}
$$




\section{HOLONOMÍA Y SU RELACIÓN ...}

se levanta dentro $\mathcal{F}_{\tilde{Z}_{1}}$ pasando por $\left(1, x_{2}\right)$ para $\left|x_{2}\right|<\rho$ en

$$
\bar{\alpha}_{x_{2}}: \mathbb{R} \rightarrow V_{\rho}, \bar{\alpha}_{x_{2}}=\left(e^{i \theta}, \alpha_{x_{2}}(\theta)\right), \text { con } \alpha_{x_{2}}(0)=x_{2},
$$

donde

$$
\begin{aligned}
\left(e^{i \theta}, \alpha_{x_{2}}(\theta)\right) & =\varphi_{i}\left(\theta,\left(1, x_{2}\right)\right)=\varphi\left(i \theta,\left(1, x_{2}\right)\right) \\
& =\left(e^{i \theta}, \varphi_{2}\left(i \theta,\left(1, x_{2}\right)\right)\right)
\end{aligned}
$$

$\Rightarrow \alpha_{x_{2}}(\theta)=\varphi_{2}\left(i \theta,\left(1, x_{2}\right)\right)$ es el flujo real de $\dot{x}_{2}=\lambda x_{2}\left(1+A\left(1, x_{2}\right)\right)$ en la dirección $i$.

Por otro lado, dentro de las hojas de $\mathcal{F}_{Z}$ el camino $\bar{\alpha}_{0}$ se levanta de la misma forma en el camino

$$
\begin{gathered}
\theta \mapsto\left(e^{i \theta}, y_{2} e^{i \lambda \theta}\right)=\left(e^{i \theta}, g_{1}\left(e^{i \theta}, y_{2}\right)\right) \\
g_{1}\left(e^{i \theta}, y_{2}\right)=y_{2} e^{i \lambda \theta},
\end{gathered}
$$

donde $\hat{\xi}\left(1, x_{2}\right)=\left(1, y_{2}\right):=\left(1, \hat{\xi}\left(x_{2}\right)\right), \hat{\xi}\left(x_{2}\right)=y_{2}$.

Sea $\hat{\xi}: V_{\rho} \rightarrow \mathbb{S}^{1} \times D_{1}$ definido por,

$$
\begin{aligned}
\tilde{\xi}\left(e^{i \theta}, \alpha_{x_{2}}(\theta)\right) & =\left(e^{i \theta}, y_{2} e^{i \lambda \theta}\right) \\
& =\left(e^{i \theta}, e^{i \lambda \theta} \hat{\xi}\left(x_{2}\right)\right), \quad \text { pues } \xi\left(x_{2}\right)=y_{2} \\
\tilde{\xi}\left(e^{i \theta}, \alpha_{x_{2}}(\theta)\right) & =\left(e^{i \theta}, g_{1}\left(e^{i \theta}, \xi\left(x_{2}\right)\right)\right),
\end{aligned}
$$

donde $g_{1}\left(e^{i \theta}, \hat{\xi}\left(x_{2}\right)\right)=e^{i \lambda \theta} \hat{\xi}\left(x_{2}\right)$ es acotado.

Observación.- $\tilde{\xi}$ es una conjugación entre hojas de $\tilde{Z}_{1}$ restringida a $\mathbb{S}^{1} \times D_{1}(0)$ con las de $Z$ restringida también a $\mathbb{S}^{1} \times D_{1}(0)$.

En efecto, sea $P=\left(x_{1}, x_{2}\right) \in \mathbb{S}^{1} \times D_{\rho}(0) \Rightarrow \exists \theta \in \mathbb{R} / \varphi\left(i \theta,\left(1, x_{2}^{\prime}\right)\right)=\left(x_{1}, x_{2}\right), x_{1}=e^{i \theta}$

$$
\Rightarrow \varphi_{2}\left(i t, \varphi\left(i \theta,\left(1, x_{2}^{\prime}\right)\right)\right)=\varphi_{2}\left(i t,\left(x_{1}, x_{2}\right)\right) \text {. }
$$

Debemos probar que,

$$
\tilde{\xi}\left(\varphi_{i}(t, P)\right)=\psi_{i}(t, \tilde{\xi}(P)) .
$$


Veamos,

$$
\begin{aligned}
\tilde{\xi}\left(\varphi_{i}\left(t, x_{1}, x_{2}\right)\right) & =\tilde{\xi}\left(\varphi\left(i t,\left(x_{1}, x_{2}\right)\right)\right)=\tilde{\xi}\left(e^{i t} x_{1}, \varphi_{2}\left(i t,\left(x_{1}, x_{2}\right)\right)\right) \\
& =\tilde{\xi}\left(e^{i t} e^{i \theta}, \varphi_{2}\left(i t, \varphi\left(i \theta,\left(1, x_{2}^{\prime}\right)\right)\right)\right. \\
& =\left(e^{i(t+\theta)}, \varphi_{2}\left(i(t+\theta),\left(1, x_{2}^{\prime}\right)\right)\right) \\
& =\left(e^{i t} e^{i \theta}, e^{i \lambda t} \hat{\xi}\left(x_{2}^{\prime}\right) e^{i \lambda \theta}\right) \\
& =\psi\left(i t,\left(e^{i \theta}, \hat{\xi}\left(x_{2}^{\prime}\right) e^{i \lambda \theta}\right)\right) \\
& =\psi(i t, \tilde{\xi}(P)) \\
& =\psi_{i}((t, \tilde{\xi}(P)))
\end{aligned}
$$

Luego,

$$
\tilde{\xi}\left(\varphi_{i}(t, P)\right)=\psi_{i}((t, \tilde{\xi}(P))), \forall P=\left(x_{1}, x_{2}\right) \in \mathbb{S}^{1} \times D_{1}(0), \forall t
$$

Asi hemos extendido

$$
\hat{\xi}:\{1\} \times D_{1}(0) \rightarrow\{1\} \times D_{1}(0)
$$

a

$$
\tilde{\xi}: \mathbb{S}^{1} \times D_{1}(0) \rightarrow \mathbb{S}^{1} \times D_{1}(0)
$$

Ahora usando levantamiento de caminos radiales, extenderemos $\tilde{\xi}$ a

$$
\xi: D_{1}(0) \times D_{1}(0) \rightarrow D_{1}(0) \times D_{1}(0) .
$$

Sea $r_{x}:[0,-\log |x|] \rightarrow L_{0}, r_{x}(t)=\left(x e^{t}, 0\right)$ para $0<|x|<1$. Por lo pronto, mostraremos que $r_{x}$ se levanta dentro de las hojas de $\mathcal{F}_{\tilde{z}_{1}}$ en $r_{x, y}: t \rightarrow\left(x e^{t}, y(t)\right) ; y(0)=y, \forall y \in D_{1},|y|<1$, es decir, que la solución $y(t)$ de la ecuación diferencial con parámetro $x, 0<|x|<1$,

$$
\frac{d y}{d t}=\lambda y(1+A(x, y))
$$

con condición inicial $y(0)=y$ es definido sobre $[0,-\log |x|]$. Luego, $\dot{y}(s)=\lambda y(s)\left(1+A\left(x e^{s}, y(s)\right)\right)$.

Entonces, $\int_{0}^{t} \frac{\dot{y}(s)}{y(s)} d s=\int_{0}^{t} \lambda\left(1+A\left(x e^{s}, y(s)\right)\right) d s$ 


\section{HOLONOMÍA Y SU RELACIÓN ...}

Luego,

$$
\begin{aligned}
& {[\log y(s)]_{0}^{t} \quad=\lambda \int_{0}^{t}\left(1+A\left(x e^{s}, y(s)\right)\right) d s} \\
& \log y(s)-\log y(0)=\lambda \int_{0}^{t}\left(1+A\left(x e^{s}, y(s)\right)\right) d s .
\end{aligned}
$$

Considerando la parte real, en ambos miembros de la igualdad anterior

$$
\begin{aligned}
\log |y(t)|-\log |y| & =\operatorname{Re}\left[\lambda \int_{0}^{t}\left(1+A\left(x e^{s}, y(s)\right)\right) d s\right] \\
& =\operatorname{Re}\left[\lambda t+\lambda \int_{0}^{t} A\left(x e^{s}, y(s)\right) d s\right]
\end{aligned}
$$

entonces

$$
\begin{aligned}
\log \left|\frac{y(t)}{y}\right| & =\lambda t+\operatorname{Re}\left[\lambda \int_{0}^{t} A\left(x e^{s}, y(s)\right) d s\right] \\
& \leq \lambda t+|\lambda| \int_{0}^{t}\left|A\left(x e^{s}, y(s)\right)\right| d s, \text { pues } \operatorname{Re}(w) \leq|w|,\left|\int f\right| \leq\left|\int f\right| \\
& \leq \lambda t+|\lambda| \frac{1}{2} \int_{0}^{t} d s, \text { pues }|A(x, y)|<\frac{1}{2} \\
& =\lambda t+|\lambda| \frac{1}{2} \\
& =\lambda t-\frac{\lambda t}{2}=\frac{\lambda t}{2}, \text { pues } \lambda \in \mathbb{R}^{-} .
\end{aligned}
$$

Aplicando el exponencial,

$$
\begin{aligned}
& \left|\frac{y(t)}{y}\right| \leq e^{\frac{\lambda t}{2}} \\
& |y(t)| \leq|y| e^{\frac{\lambda t}{2}} \leq|y|<1, \forall t \in[0,-\log |x|] . \quad \text { Si } t \rightarrow \infty,|y(t)| \rightarrow 0 .
\end{aligned}
$$

En el resultado anterior, usando las notaciones convenientemente (por estar en el campo $\tilde{Z}_{1}$ ) se deben cambiar $x$ por $x_{1}$ e $y$ por $x_{2}$. Con ello se tendrá que si $t \rightarrow \infty$ (cuando $x \rightarrow 0$ ), entonces $\left|x_{2}(t)\right| \rightarrow 0$. Luego el conjunto $V$ de los puntos $\left(x_{1}, x_{2}\right) \in U\left(x_{1} \neq 0\right)$ vecindad de $0 \in \mathbb{C}^{2}$, tales que el extremo de $r_{x, y} \in V_{\rho}$ es un abierto y $V \cup\left\{x_{1}=0\right\}$ es una vecindad de 0 dentro de $\mathbb{C}^{2}$.

Por otro lado, el camino inverso de $r_{x}$

$$
r_{x}^{-1}: t \mapsto\left(x e^{-t-\log |x|}, 0\right)
$$


se levanta dentro de las hojas de $\mathcal{F}_{Z}$ pasando por el punto $\left(\frac{x}{|x|}, y_{2}\right)$ en un camino cuyo punto inicial es $\left(\frac{x}{|x|}, y_{2}\right)$ y el extremo $\left(x, e^{-\lambda \tau} y_{2}\right), \tau=-\log |x|$, es decir que, la solución $y_{2}(t)$ de la ecuación diferencial $\frac{d y_{2}}{d t}=\lambda y_{2}$, con $y_{2}(0)=y_{2}$ es definido sobre $[0,-\log |x|]$. Luego

$$
\dot{y}_{2}(s)=\lambda y_{2}(s) \Rightarrow y_{2}(t)=e^{\lambda t} y_{2}, \forall t \in[0, \log |x|]
$$

Vemos que si $t \rightarrow \infty,\left|y_{2}(t)\right| \rightarrow 0$, es decir, $\left|y_{2}(t)\right|$ es acotado, $\forall t \in[0,-\log |x|]$. Asi entonces $\xi$, la aplicación de $V$ dentro de $\mathbb{C}^{2}$ definido por

$$
\left(x_{1}, x_{2}\right) \mapsto\left(x_{1}, e^{\lambda\left(\log \left|x_{1}\right|\right)} y_{2}\right)
$$

donde

$$
\tilde{\xi}\left(\frac{x}{|x|}, x_{2}(\tau)\right)=\left(\frac{x}{|x|}, y_{2}\right) \Rightarrow y_{2}=\tilde{\xi}_{2}\left(\frac{x}{|x|}, x_{2}(\tau)\right) .
$$

Asi,

$$
\begin{aligned}
\xi\left(x_{1}, x_{2}\right) & =\left(x_{1}, e^{\lambda\left(\log \left|x_{1}\right|\right.} \tilde{\xi}_{2}\left(\frac{x}{|x|}, x_{2}(\tau)\right)\right) \\
& =\left(x_{1}, e^{\lambda\left(\log \left|x_{1}\right|\right.} \tilde{\xi}_{2}\left(\frac{x}{|x|}, \varphi_{2}\left(-\log \left|x_{1}\right|,\left(x_{1}, x_{2}\right)\right)\right)\right)
\end{aligned}
$$

o también,

$$
\xi\left(x_{1}, x_{2}\right)=\left(x_{1}, g\left(x_{1}, x_{2}\right)\right)
$$

donde

$$
g\left(x_{1}, x_{2}\right)=\left|x_{1}\right|^{\lambda} \tilde{\xi}_{2}\left(\frac{x_{1}}{\left|x_{1}\right|}, \varphi_{2}\left(-\log \left|x_{1}\right|,\left(x_{1}, x_{2}\right)\right)\right)
$$

además vemos que $g\left(x_{1}, x_{2}\right)$ es acotado. Luego, $\xi$ es acotado (pues sus funciones componentes lo son), también es holomorfa y se puede ver además que $\xi$ es una conjugación de las hojas $\mathcal{F}_{\tilde{z}_{1} \mid V}$ y $\mathcal{F}_{Z \mid \xi(V)}$.

Para verificar la última afirmación debemos probar que

$$
\xi\left(\varphi\left(T, x_{1}, x_{2}\right)\right)=\psi\left(T, \xi\left(x_{1}, x_{2}\right)\right)
$$




\section{HOLONOMÍA Y SU RELACIÓN ...}

tenemos que,

$$
\begin{aligned}
\xi\left(\varphi\left(T, x_{1}, x_{2}\right)\right) & =\xi\left(e^{T} x_{1}, \varphi_{2}\left(T, x_{1}, x_{2}\right)\right) \\
& =\left(e^{T} x_{1}, e^{\lambda\left(\log \left|e^{T} x_{1}\right|\right)} \tilde{\xi}_{2}\left(\frac{e^{T} x_{1}}{\left|e^{T} x_{1}\right|}, \varphi_{2}\left(-\log \left|e^{T} x_{1}\right|,\left(e^{T} x_{1}, \varphi_{2}\left(T, x_{1}, x_{2}\right)\right)\right)\right) .\right.
\end{aligned}
$$

Si consideramos $T=t+\theta i \Rightarrow-\log \left|e^{T} x_{1}\right|=-\log \left|e^{T}\right|\left|x_{1}\right|=-t-\log \left|x_{1}\right|$, entonces

$$
\begin{aligned}
& \xi\left(\varphi\left(T, x_{1}, x_{2}\right)\right)=\left(e^{T} x_{1}, e^{\lambda t+\lambda \log \left|x_{1}\right|} \tilde{\xi}_{2}\left(\frac{e^{i \theta} x_{1}}{\left|x_{1}\right|}, \varphi_{2}\left(-\log \left|e^{T} x_{1}\right|, e^{T} x_{1}, \varphi_{2}\left(T, x_{1}, x_{2}\right)\right)\right)\right) \\
& =\left(e^{T} x_{1}, e^{\lambda t}\left|x_{1}\right|^{\lambda} \tilde{\xi}_{2}\left(\frac{e^{i \theta} x_{1}}{\left|x_{1}\right|}, \varphi_{2}\left(-t-\log \left|x_{1}\right|, \varphi\left(T, x_{1}, x_{2}\right)\right)\right)\right) \\
& =\left(e^{T} x_{1}, e^{\lambda t}\left|x_{1}\right|^{\lambda} \tilde{\xi}_{2}\left(\frac{e^{i \theta} x_{1}}{\left|x_{1}\right|}, \varphi_{2}\left(-t-\log \left|x_{1}\right|+t+i \theta,\left(x_{1}, x_{2}\right)\right)\right)\right) \\
& =\left(e^{T} x_{1}, e^{\lambda t}\left|x_{1}\right|^{\lambda} \tilde{\xi}_{2}\left(\frac{e^{i \theta} x_{1}}{\left|x_{1}\right|}, \varphi_{2}\left(-\log \left|x_{1}\right|+i \theta ;\left(x_{1}, x_{2}\right)\right)\right)\right) \\
& =\left(e^{T} x_{1}, e^{\lambda t}\left|x_{1}\right|^{\lambda} \tilde{\xi}_{2}\left(\frac{e^{i \theta} x_{1}}{\left|x_{1}\right|}, \varphi_{2}\left(i \theta, \varphi\left(-\log \left|x_{1}\right|,\left(x_{1}, x_{2}\right)\right)\right)\right)\right. \\
& =\left(e^{T} x_{1}, e^{\lambda t}\left|x_{1}\right|^{\lambda} \tilde{\xi}_{2}\left(\varphi\left(i \theta, \frac{x_{1}}{\left|x_{1}\right|}, \varphi_{2}\left(-\log \left|x_{1}\right|,\left(x_{1}, x_{2}\right)\right)\right)\right)\right) \\
& =\left(e^{T} x_{1}, e^{\lambda t}\left|x_{1}\right|^{\lambda} \psi_{2}\left(i \theta, \tilde{\xi}\left(\frac{x_{1}}{\left|x_{1}\right|}, \varphi_{2}\left(-\log \left|x_{1}\right|,\left(x_{1}, x_{2}\right)\right)\right)\right)\right) \\
& =\left(e^{T} x_{1}, e^{\lambda t}\left|x_{1}\right|^{\lambda} e^{i \lambda \theta} \tilde{\xi}_{2}\left(\frac{x_{1}}{\left|x_{1}\right|}, \varphi_{2}\left(-\log \left|x_{1}\right|,\left(x_{1}, x_{2}\right)\right)\right)\right) \\
& =\left(e^{T} x_{1}, e^{\lambda t} e^{i \lambda \theta} e^{\left.\lambda \log \left|x_{1}\right| \tilde{\xi}_{2}\left(\frac{x_{1}}{\left|x_{1}\right|}, \varphi_{2}\left(-\log \left|x_{1}\right|,\left(x_{1}, x_{2}\right)\right)\right)\right)}\right. \\
& =\left(e^{T} x_{1}, e^{\lambda t} e^{i \lambda \theta} \tilde{\xi}_{2}\left(x_{1}, x_{2}\right)\right) \\
& =\left(e^{T} \xi_{1}\left(x_{1}, x_{2}\right), e^{\lambda T} \xi_{2}\left(x_{1}, x_{2}\right)\right) \\
& =\psi\left(T, \xi\left(x_{1}, x_{2}\right)\right) \text {. }
\end{aligned}
$$

Así hemos probado que

$$
\xi\left(\varphi\left(T, x_{1}, x_{2}\right)\right)=\psi\left(T, \xi\left(x_{1}, x_{2}\right)\right),
$$

Luego, por el teorema de extensión de Riemann (Gunning - Rossi [5]), $\xi$ se extiende a $V \cup\{x=0\}$. Asi entonces, existe un difeomorfismo local de $\mathbb{C}^{2}$ en $(0,0) \in \mathbb{C}^{2}$ que transforma la foliación asociada a $\tilde{Z}_{1}=\left(x_{1}, x_{2} \lambda\left(1+A\left(x_{1}, x_{2}\right)\right)\right)$ en aquella asociada a $Z=\left(y_{1}, \lambda y_{2}\right)$. 
Sean ahora dos campos no lineales $W_{1} y W_{2}$ con

$$
W_{j}=\left(\alpha_{1}^{j} x_{1}+x_{1} x_{2} \alpha_{1}^{j}\left(x_{1}, x_{2}\right), \alpha_{2}^{j} x_{2}+x_{1} x_{2} \alpha_{2}^{j}\left(x_{1}, x_{2}\right)\right),
$$

entonces dos campos no lineales son conjugados, si existe $\hat{\xi}$ difeomorfismo local de $0 \in \mathbb{C}$ tal que, $h_{2} \circ \hat{\xi}=\hat{\xi} \circ h_{1}$, donde $h_{j}(j=1,2)$ son las holonomias asociadas a $W_{1}$ y $W_{2}$ respectivamente en $(1,0)$.

\section{CONCLUSIONES}

Las holonomias $h: \mathbb{C} \rightarrow \mathbb{C}$ consideradas en la sección anterior tiene la característica de que

$$
h^{\prime}(0)=e^{2 \pi \lambda i}, \lambda \in \mathbb{C} .
$$

Además tienen otras propiedades las cuales nos informan si el campo vectorial es linealizable o no. La justificación de éstos hechos será motivo de un posterior estudio.

\section{REFERENCIAS BIBLIOGRÁFICAS}

[1] Benazic, R. Singularidades de Campos Vectoriales Holomorfos en el Dominio de Poincaré, Pro-Matemática, Vol X, No 19-20 Lima (1996).

[2] Camacho, C. Holomorphic Dynamical Systems; Summer School on Dynamical Systems ( 1 st -25 th august 1983) triestre P.O.B. 586 ITALY.

[3] Camacho, C; Sad, P. Pontos Singulares de Equações Diferenciais Analíticas, 16 Coloquio Brasileiro de Matemática, IMPA, (1987).

[4] Hirsch, M; Smale, S. Differential Equations, Dynamical Systems and Linear Algebra, Academic Press, New York, (1974).

[5] Gunning, Rossi H. Analytic Functions of Several Variables, Prentice Hall (1995).

[6] Mattei, J; Moussu, R. Holonimie et Integrales Premieres, Ann. Scie. Ec. Norm. Sup. (4) 13 (1980) pag. 469-523.

[7] Ramírez, S. Tópicos de Teoría Cualitativa de Ecuaciones Diferenciales Ordinarias, Tesis Licenciatura, UNMSM, (2001).

[8] Sotomayor, J. Lições de Equações Diferenciais Ordinarias, Projeto Euclides (1979). 